\title{
Recettes de macérâts huileux : intérêt pour la cosmétique
}

Fabrice TURON

Michel Bertin SA,

Route de Mareuil,

BP 51, 60330 Lagny le Sec

Fax : 0344608076

<huiles-michelbertin@wanadoo.fr>

\section{Principes de la macération}

Un macérât huileux est un principe ancien et facile à mettre en œuvre. Il consiste à mélanger des extraits végétaux dans un corps gras qui, jouant le rôle de solvant naturel, permet d'extraire par un simple pressage les principes actifs liposolubles. Par contre, le choix du corps gras de concentration est essentiel. En effet, le corps gras est sélectionné en fonction de ses propriétés intrinsèques, c'est-à-dire sa composition en acides gras ou son insaponifiable, qu'il peut ajouter à celles du végétal traité [1]. Ainsi, une macération au soleil de millepertuis (Hypericum perforatum) dans de I'huile d'olive permet à cette dernière de $s^{\prime}$ enrichir en agents anti-inflammatoires du millepertuis. Le mélange final, qui combine les bienfaits de I'olive et du millepertuis, est recommandé dans le traitement topique des brûlures $[2,3]$. Toutefois, le fait que la macération au soleil dure plusieurs semaines tend à altérer la qualité de I'huile d'olive. C'est pourquoi la durée d'imprégnation de l'extrait végétal par le corps gras est aujourd'hui considérablement réduite à quelques heures en chauffant le mélange à l'étuve, en atmosphère inerte pour éviter l'oxydation.

\section{Utilisations en cosmétique}

Les macérâts huileux sont couramment utilisés dans des préparations cosmétiques. En détaillant quelques recettes, nous nous proposons ici de démontrer leurs intérêts.

\section{Parfumer une huile}

D'abord, les macérâts huileux sont depuis longtemps utilisés pour parfumer des corps gras. En Haute-Egypte, on mélangeait des fleurs fraîchement cueillies à du suif (ou du saindoux) bouillant pour solubiliser les essences de parfum. En 1860, les huiles essentielles sont piégées selon le même principe dans un corps gras, dénommé "pommade», pour ensuite être extraites par distillation alcoolique.

\begin{abstract}
The maceration is a particular contact process between virgin oil (as a solvent) and seeds, leaves or plant extracts (as a solid). This process enables the extraction of liposoluble content of the solid by ordinary pressure, using the virgin oil as a natural solvent. Moreover, it allows the extraction of liposoluble materials which are hardly extractable by simple pressure. In this review, traditional macerates and their cosmetic uses are reported.
\end{abstract}

Key words: maceration, oil, tocopherol, essential fatty acids

À titre d'information, ce savoir-faire a fait I'objet d'un brevet américain déposé en 2002 [4]. En Polynésie, le monoï ou littéralement " huile parfumée », est issu de fleurs de tiare récoltées au stade de boutons, qui sont mises en macération dans de l'huile de coco pendant au moins deux semaines. L'appellation d'origine du monoï n'est accordée que si ces fleurs sont cueillies sur le territoire polynésien et si les proportions et délais de macération sont respectés.

\section{Colorer une huile}

Les macérâts huileux permettent encore de colorer naturellement une huile. En considérant du germe de blé en macération dans de I'huile vierge d'amandes douces, on obtient un produit qui, outre son enrichissement en tocophérols du germe de blé, va se colorer de jaune à oranger (figure 1). Un deuxième exemple consiste à faire macérer du curcumin (Curcuma longa) dans de I'huile de noisettes; ce mélange va se colorer en jaune soleil par un transfert des curcuminoïdes dans I'huile. Dans la figure 2, l'absorbance de I'huile de noisettes « curcumisée » est mesurée dans I'UV. Les deux pics principaux observés à 245 et $430 \mathrm{~nm}$ correspondent aux maximum d'absorption du curcumin. Rappelons que, outre sa couleur, le curcumin est connu pour ses activités antiinflammatoire et antioxydante [5-7].

\section{Réguler l'inflammation cutanée}

L'inflammation au niveau de la peau implique certains médiateurs biologiques dont le lien commun est l'acide arachidonique, présent dans la couche basale au niveau de la jonction dermo-épidermique. Tant que l'acide arachidonique est lié à un phospholipide, il est stable. Mais en cas d'altération de la peau (vieillissement, détergent, UV), il est libéré sous I'action $d^{\prime}$ une phospholipase $A 2$, en particulier pour donner naissance à un métabolite proinflammatoire, le leucotriène LTB4 $[8,9]$. On connaît un monohydroxyacide capable d'inhi- ber la production de LTB4: c'est le 15-hydroxyeicosapentaénoïque (15 HEPE), qui est métabolisé dans l'épiderme par la 15-lipoxygénase à partir de l'acide eicosapentaénoïque (EPA, 20:5 n-3). Les sources naturelles de l'EPA sont les huiles de poisson et certaines algues. Deux algues en particulier vont intéresser la suite de notre propos: ce sont Ascophyllum nodosum, de couleur verte à orangée, qui est récoltée de décembre à mars, période pendant laquelle les réceptacles se détachent de l'algue, et dont la teneur en lipides est de l'ordre de $5 \%$, et Undaria pinnatifida, algue brune autrement appelée «fougère de mer ", dont la teneur en lipides est inférieure à $3 \%$. Ces algues présentent une teneur en EPA respectivement de 20 et 14 $\mathrm{mg} / \mathrm{g}$ d'extrait lipidique sec. Même si ces teneurs en EPA sont intéressantes, l'extraction directe de l'huile est économiquement impossible au regard des trop faibles teneurs en lipides totaux. En considérant maintenant une huile de noisette obtenue après macération dans ces algues, on constate dans la figure 3 qu'elle s'enrichit en EPA de manière quantitativement plus importante avec Undaria (20 $\mathrm{mg} / \mathrm{g}$ ) comparée à Ascophyllum (4 mg/g). Dans tous les cas, les huiles obtenues sont colorées et dégagent une odeur marine marquée.

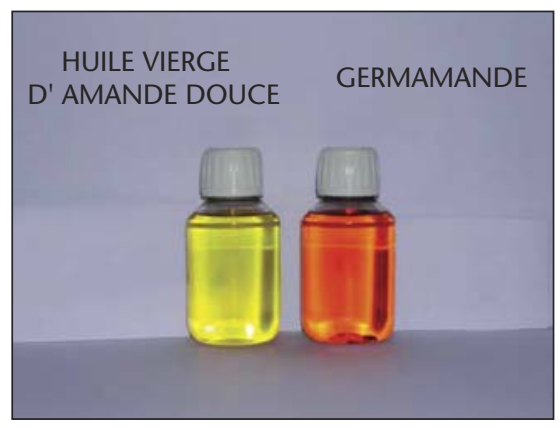

Figure 1. Exemple de coloration d'une huile vierge d'amande douce après macération dans du germe de blé (Germamande). 


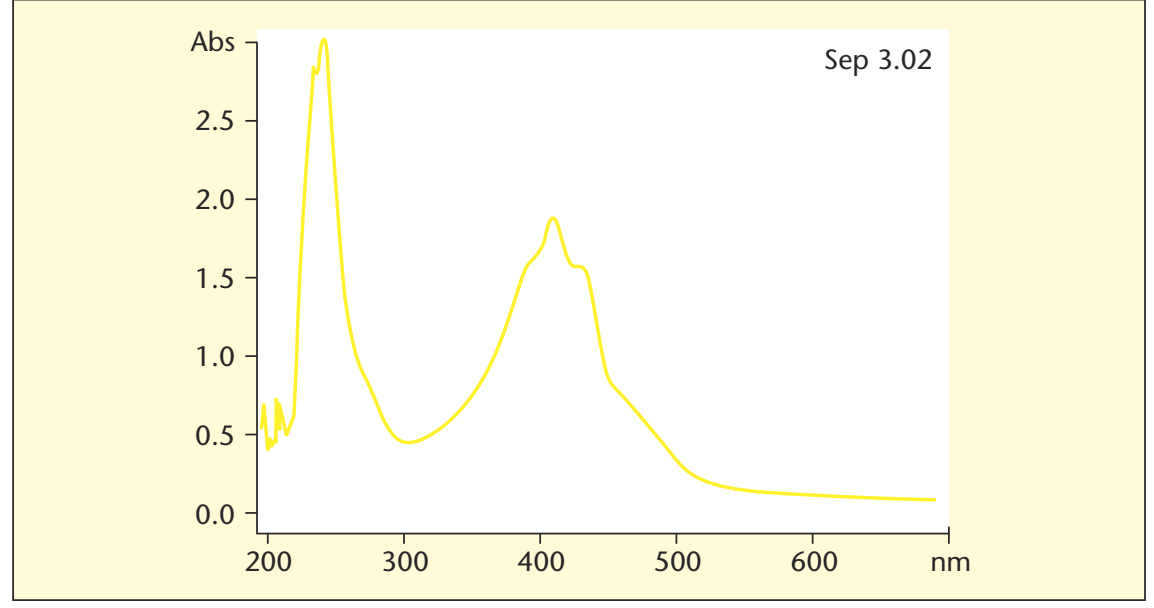

Figure 2. Spectre UV de I'huile de noisettes après macération dans du curcumin.

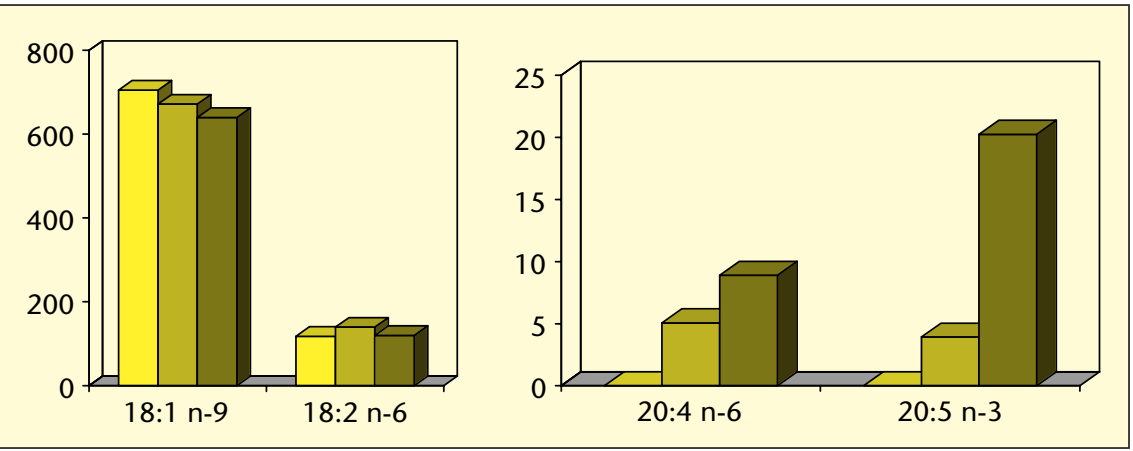

Figure 3. Composition en principaux acides gras (en $\mathrm{mg} / \mathrm{g}$ ) de macérâts d'algues. De gauche à droite : huile vierge de noisettes, macérât noisettes/Ascophyllum, macérât noisettes/Undaria.

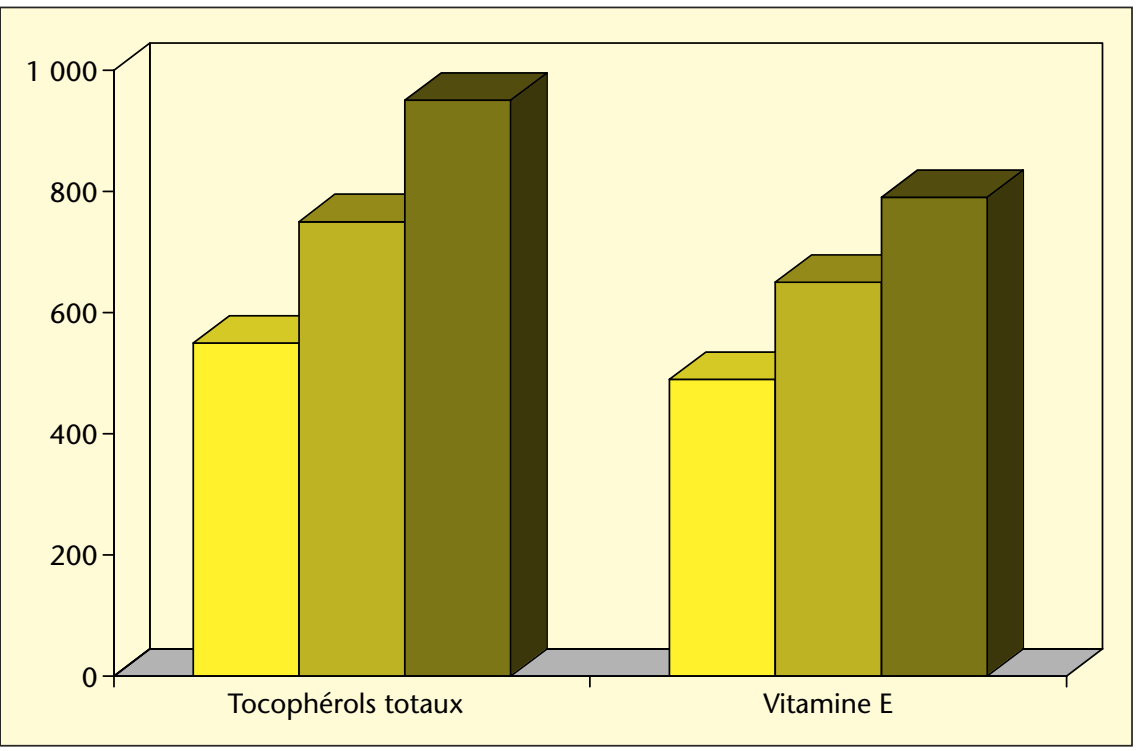

Figure 4. Composition en tocophérols totaux et $\alpha$ tocophérols (en $\mathrm{mg} / \mathrm{kg}$ ) des macérâts huileux de quinoa (Hélioquinoa) et de germe de blé (Héliogerm) dans de l'huile vierge de tournesol. De gauche à droite : huile vierge de tournesol, Hélioquinoa, Héliogerm.

\section{Enrichir en vitamine $E$}

Un macérât huileux permet d'enrichir une huile en insaponifiable. La quinoa, Chenopodium quinoa, est une plante cultivée sur les hauts plateaux Andins. A l'origine, elle était une nourriture de base des Incas, connue sous son nom Quechua de chisiya mama, qui peut se traduire par « graine nourricière ». Elle disparut avec la conquête de l'Amérique du Sud par les Espagnols au XV' siècle et l'introduction dans la région de leur propre alimentation. Le contenu en lipides de la graine est de l'ordre de $6 \%$, mais surtout sa teneur en vitamine $E$ est remarquablement élevée (54 mg/kg matière sèche) comparativement au germe de blé $(12 \mathrm{mg} / \mathrm{kg}$ ) $[10,11]$. Des études de macération de ces deux graines ont été réalisées pour comparer le transfert effectif des tocophérols totaux, en particulier de la vitamine E, dans de I'huile vierge de tournesol. La figure 4 illustre une teneur en vitamine $E$ du macérât plus importante avec le germe de blé $(790 \pm 100 \mathrm{mg} / \mathrm{kg}$ huile) que la quinoa $(650 \pm 100 \mathrm{mg} / \mathrm{kg})$. Cette observation suggère que le transfert des composés liposolubles n'est pas seulement dépendant de leur concentration dans la matrice végétale. D'autres critères, comme leur accessibilité ou la polarité de I'huile d'extraction, sont certainement à prendre en considération.

En conclusion, on retiendra des macérâts huileux qu'ils permettent d'extraire naturellement le contenu lipidique d'une graine ou d'une plante jugé trop faible pour une extraction économiquement rentable, et que I'huile de concentration, écologiquement propre, remplace un solvant usuel d'extraction comme I'hexane.

\section{RÉFÉRENCES}

1. LE CLERC MJ. Procédé de préparation de produits de beauté à bases végétales actives. Bulletin officiel de la Propriété Industrielle 1966; 6 ( $n^{\circ}$ 3) : 714 .

2. MAISENBACHER P, KOVAR KA. Analysis and stability of Hyperici Oleum. Planta Med 1992 ; $58: 351-4$.

3. SCHEMPP CM, MULLER KA, WINGHOFER B, SCHOPF E, SIMON JC. St. John's wort (Hypericum perforatum L.). A plant with relevance for dermatology. Hautarzt $2002 ; 53: 316-21$.

4. JUNGHANNS W, SCHIERSTEDT G, GRZESCHIK E, PIELA R. Process of producing enriched vegetable oils. US Patent Application Publication $2002\left(n^{\circ} 09 / 917\right) ; 380$. 
5. THRESIAMMA KC, GEORGE J, KUTTAN R. Protective effect of curcumin, ellagic acid and bixin on radiation induced genotoxicity. I Exp Clin Res $1998 ; 17: 431-4$.

6. RUKKUMANI R, BALASUBASHINI MS, MENON VP. Protective effects of curcumin and photoirradiated curcumin on circulatory lipids and lipid peroxidation products in alcohol and polyunsaturated fatty acid-induced toxicity. Phytother Res $2003 ; 17$ : 925-9.
7. SHIPOCHLIEV T, DIMITROV A, ALEKSANDROVA E. Anti-inflammatory action of a group of plant extracts. Vet Med Nauki 1981; 18 : 87-94.

8. HORROBIN DF. The regulation of prostaglandin biosynthesis by manipulation of essential fatty acid metabolism. Rev Pure Appl Pharm Sci $1983 ; 4: 339-83$

9. BRAIN S, CAMPR, DOWD P. Psoriasis and leukotriene B4. Lancet $1983 ; 2$ : 762-3.
10. WOOD SG, LAWSON LD, FAIRBANKS DJ, ROBISON LR, ANDERSEN WR. Seed lipid content and fatty acid composition of three quinoa cultivars. / Food Composition Analysis 1993; 6 : 41-4.

11. PRZYBYLSKI R, CHAUHAN GS, ESKIN NAM. Characterization of quinoa (Chenopodium quinoa) lipids. Food Chem $1994 ; 51$ : 187-92. 